DESY-99-065

TTP99-26

June 1999

\title{
Spin Dependence of Deep Inelastic Diffraction
}

\author{
J. Bartels ${ }^{a}$, T. Gehrmann ${ }^{b}$ and M.G. Ryskin ${ }^{c}$ \\ ${ }^{a}$ II. Institut für Theoretische Physik, Universität Hamburg, D-22761 Hamburg, Germany \\ ${ }^{b}$ Institut für Theoretische Teilchenphysik, Universität Karlsruhe, D-76128 Karlsruhe, \\ Germany \\ ${ }^{c}$ Petersburg Nuclear Physics Institute, 188350 Gatchina, St. Petersburg, Russia
}

\begin{abstract}
Using a perturbative model for diffractive interactions, we derive an expression for the polarized diffractive structure function $g_{1}^{D}$ in the high energy limit. This structure function is given by the interference of diffractive amplitudes with polarized and unpolarized exchanges. For the polarized exchange we consider both twogluon and quark-antiquark amplitudes. The polarized diffractive amplitude receives sizable contributions from non-strongly ordered regions in phase space, resulting in a double logarithmic enhancement at small $x$. The resummation of these double logarithmic terms is outlined. We also discuss the transition from our perturbative expression to the nonperturbative region. A first numerical estimate indicates that the perturbative contribution to the spin asymmetry is substantially larger than the nonperturbative one.
\end{abstract}




\section{Introduction}

The occurrence of large rapidity gaps between current jet direction and proton remnant direction in electron-proton collisions at HERA represents one of the most puzzling phenomena in the physics of deep inelastic scattering. The first observation of these diffractive deep inelastic scattering events has triggered much theoretical effort towards gaining an understanding of this phenomenon. Although much progress both in the theoretical description and in the experimental study of diffraction in deep inelastic scattering has been made, it is still fair to say that this phenomenon is not unambiguously understood at present, since it contains both perturbative and nonperturbative components.

To summarize our present understanding of (unpolarized) diffraction in deep inelastic scattering, the diffractive final states can be attributed either to nonperturbative soft exchanges or to hard exchanges, represented by the (slightly nonforward) gluon structure function. Examples of the former class are the aligned jet configuration of diffractively produced quark-antiquark pairs or the analogous quark-antiquark gluon configuration where the gluon is rather soft (in particular, has a small transverse momentum). For the latter class of final states, we mention the diffractive production of longitudinal vector particles or quark-antiquark jets with large transverse momenta. One of the most prominent signatures of these distinct classes is the energy dependence: for the nonperturbative part one expects to see the energy dependence of the soft Pomeron, whereas the perturbative part should be characterized by the stronger increase with energy as observed in the gluon structure function at small $x$, as determined by perturbative QCD. The diffractive contribution to deep inelastic structure functions can not be easily attributed to one of both classes. Experimental data on it lie indeed between the two extremes, thus indicating the existence of sizable contributions from both hard and soft exchanges.

The currently discussed option of operating the HERA collider with a polarized proton beam naturally leads to the expectation that, again, DIS diffraction will be an important phenomenon, and hard and soft physics will compete with each other. In particular, one might expect that, as in the unpolarized case, a substantial part of the diffractive cross section may be calculable within perturbative QCD.

Recently [1] a detailed study of the non-perturbative contributions to spin asymmetries in deep inelastic diffraction has lead to the conclusion that these are very small. The investigation has been carried out in the framework of Regge theory: the amplitude for diffraction is described by Pomeron pole exchange and has a (small) spin-dependent component. It was found that this amplitude alone yields a vanishing spin asymmetry. Non-zero asymmetries can be obtained only if, in addition to the simple Pomeron pole, also secondary Reggeon exchanges $\left(\rho, \omega, f, A_{2}\right)$ as well as multi-Pomeron and PomeronReggeon cuts are taken into account. Contributions due to multi-Pomeron cuts turn out to be negligible small. The dominant contribution to diffractive spin asymmetries arises from the interference of the amplitudes for Pomeron-Reggeon and single Reggeon exchange. As a result, the polarized cross section is suppressed, in comparison with the unpolarized cross section, by one inverse power of the collision energy. Another nonzero contribution is due to the exchange of unnatural parity $\left(\pi, a_{1}\right)$ which, again, is suppressed by one inverse power of energy. The numerical study of all these contributions shows 
that, in addition to the energy-dependent suppression, they also come with very small coefficients. In total, the resulting nonperturbative contribution to the spin asymmetry does not exceed $10^{-4}$ [1].

This raises the interesting possibility that, unlike in the unpolarized case where the nonperturbative contributions are not small, polarized diffraction may be dominated by the perturbative component. In perturbative QCD the diffractive exchange is modelled by the (slightly nonforward) unpolarized gluon structure function $g\left(x, \mu^{2}\right)$, whereas for the polarized part one uses the polarized gluon density $\Delta g\left(x, \mu^{2}\right)$ and quark density $\Delta q\left(x, \mu^{2}\right)$. In this framework the spin asymmetry is then described by the interference of the unpolarized and polarized diffractive amplitudes. Since at small $x$ the polarized quark and gluon densities - apart from logarithmic corrections - are known to be suppressed by one power of energy compared to the unpolarized gluon density, the asymmetry, when calculated perturbatively, has the same energy dependence as the nonperturbative contributions obtained from Regge theory [1]. An important enhancement of the perturbative contributions, however, is due to the logarithmic corrections which lead to a strong rise of the polarized gluon distribution at small $x$. In [2] it has been shown that, unlike the unpolarized case, the polarized structure functions have double logarithms in $1 / x$, which result in a substantial enhancement of the polarized distributions. Applied to diffractive phenomena, this enhancement implies that the ratio of perturbative to non-perturbative contributions could be much more favourable in the polarized case than in the unpolarized case.

In the present paper, we investigate the perturbative contribution to spin asymmetries in deep inelastic scattering by computing the longitudinal spin-spin asymmetry for the diffractive production of light quark-antiquark pairs. For the polarized exchange we include both two-gluon and quark-antiquark exchange. Basic features of the spin dependent cross section are first elaborated for a quark target. We demonstrate that the spin dependent part of the amplitude receives an essential contribution from a non-strongly ordered region, where the transverse momenta of the scattered quarks are lower than transverse momenta occurring in the exchange system. These contributions give rise to double logarithmic terms. Including the double logarithmic results for the polarized amplitudes from [2] we obtain a compact expression for the perturbative contribution to the asymmetry. Precise numerical predictions of this asymmetry would require knowledge on the behaviour of polarized quark and gluon distributions at small $x$, which are only poorly determined at present. A rough estimation of the perturbative contribution does however indicate the resulting asymmetry to be substantially larger than the nonperturbative contributions computed in [1].

\section{Kinematics and basic formulae}

Cross sections for diffractive DIS are defined in close analogy to the cross sections in inclusive DIS. A summary of formulae for the inclusive case can for example be found in [3]. Since we want to define a consistent framework for spin asymmetries in diffractive DIS, let us briefly review the conventionally used definitions for polarized cross sections 
and asymmetries.

In lepton-nucleon scattering with longitudinally polarized lepton beam and nucleon target, one can choose four different combinations of beam and target polarizations. If only parity conserving interactions are relevant, these can be expressed in terms of two independent cross sections:

$$
\mathrm{d} \Delta \sigma \equiv\left(\mathrm{d} \sigma^{\stackrel{\rightleftarrows}{\rightleftarrows}}-\mathrm{d} \sigma^{\rightrightarrows}\right), \quad \mathrm{d} \bar{\sigma} \equiv \frac{1}{2}\left(\mathrm{~d} \sigma^{\stackrel{\rightleftarrows}{\rightleftarrows}}+\mathrm{d} \sigma^{\rightrightarrows}\right),
$$

where the arrows denote the spin directions of beam and target. Using these, one obtains the longitudinal spin asymmetry

$$
A_{L}=\frac{\Delta \sigma}{2 \bar{\sigma}} .
$$

The above cross sections define the unpolarized and polarized structure functions as described in [3].

Processes in deep inelastic scattering can be conveniently described as product of a virtual photon flux factor and a reduced photon-proton cross section. Unpolarized and polarized cross sections at small $x$ read then:

$$
\begin{aligned}
\frac{\mathrm{d} \sigma}{\mathrm{d} x \mathrm{~d} Q^{2}} & =\frac{\alpha_{e m}}{\pi x Q^{2}}\left(1-y+\frac{y^{2}}{2}\right) \sigma_{T}^{\gamma^{*} p}, \\
\frac{\mathrm{d} \Delta \sigma}{\mathrm{d} x \mathrm{~d} Q^{2}} & =\frac{\alpha_{e m}}{\pi x Q^{2}}\left(y-\frac{y^{2}}{2}\right) \Delta \sigma^{\gamma^{*} p} .
\end{aligned}
$$

In the expression for the unpolarized cross section, we have restricted ourselves to the (dominant) contribution $\sigma_{T}^{\gamma^{*} p}$ from the average over the two transverse photon polarizations. The full unpolarized deep inelastic cross section is obtained by adding also the contribution from longitudinal photon polarization. The polarized photon-proton cross section $\Delta \sigma^{\gamma^{*} p}$ corresponds to the difference of the two transverse polarizations of the virtual photon.

The kinematics of the diffractive reaction

$$
e(l) p(p) \longrightarrow e\left(l^{\prime}\right) p\left(p^{\prime}\right) X\left(p_{X}\right)
$$

are described by the invariants

$s=(l+p)^{2}, \quad Q^{2}=-\left(l-l^{\prime}\right)^{2}, \quad \hat{s}=\left(l-l^{\prime}+p\right)^{2}, \quad M^{2}=p_{X}^{2}, \quad t=\left(p-p^{\prime}\right)^{2}$.

From these, we define the commonly used dimensionless parameters

$$
x=Q^{2} / \hat{s}, \quad y=\hat{s} / s, \quad \beta=Q^{2} /\left(Q^{2}+M^{2}\right), \quad x_{I P}=x / \beta .
$$

Using these variables, one can express the cross section for unpolarized diffraction in terms of the diffractive deep inelastic structure function $F_{T}^{D}$ :

$$
\frac{\mathrm{d} \sigma}{\mathrm{d} \beta \mathrm{d} Q^{2} \mathrm{~d} x_{I P} \mathrm{~d} t}=\frac{4 \pi \alpha_{e m}^{2}}{\beta Q^{4}}\left(1-y+\frac{y^{2}}{2}\right) F_{T}^{D}\left(\beta, Q^{2}, x_{I P}, t\right) .
$$


Analogously, we define the polarized diffractive structure function $g_{1}^{D}$ by

$$
\frac{\mathrm{d} \Delta \sigma}{\mathrm{d} \beta \mathrm{d} Q^{2} \mathrm{~d} x_{I P} \mathrm{~d} t}=\frac{16 \pi \alpha_{e m}^{2}}{Q^{4}}\left(y-\frac{y^{2}}{2}\right) g_{1}^{D}\left(\beta, Q^{2}, x_{I P}, t\right) .
$$

In the following section, we will derive $g_{1}^{D}$ in the framework of a perturbative model for diffractive reactions. Within the same model, $F_{T}^{D}$ was derived in various places in the literature [4, 5]. To illustrate similarities and differences between unpolarized and polarized calculation, we will discuss $g_{1}^{D}$ and $F_{T}^{D}$ in parallel below.

\section{Calculation of $g_{1}^{D}$}

In a perturbative framework, hard diffraction is described by the exchange of two partons in a color singlet state between the target hadron and a partonic fluctuation of the incoming virtual photon, which is converted into a diffractive system of mass $M$. The corresponding partonic reaction reads:

$$
\gamma^{*}(q) p(p) \longrightarrow q\left(k_{q}\right) \bar{q}\left(k_{\bar{q}}\right) p\left(p^{\prime}\right)
$$

We use a Sudakov-parametrization with an auxiliary vector $q^{\prime}=q+(x / \hat{s}) p$ to describe the partonic momenta in this reaction

$$
\begin{aligned}
& k_{q}=\alpha q^{\prime}+\frac{k_{t}^{2}}{\alpha \hat{s}}+\overrightarrow{k_{t}}, \\
& k_{\bar{q}}=(1-\alpha) q^{\prime}+\frac{k_{t}^{2}}{(1-\alpha) \hat{s}}-\overrightarrow{k_{t}}, \\
& l_{n}=\alpha_{n} q^{\prime}+\beta_{n} p+\overrightarrow{l_{t, n}} .
\end{aligned}
$$

The integral over the exchange loop momentum reads in these parameters

$$
\mathrm{d}^{4} l_{n}=\frac{\hat{s}}{2} \mathrm{~d} \alpha_{n} \mathrm{~d} \beta_{n} \mathrm{~d}^{2} l_{t, n}
$$

The corresponding Feynman diagrams for the two gluon exchange and quark/antiquark exchange amplitudes are depicted in Fig. 1. The cross section for this process reads

$$
\frac{\mathrm{d} \Delta \sigma^{\gamma^{*} p}}{\mathrm{~d} t \mathrm{~d} M^{2}}=\frac{1}{(4 \pi)^{4}} \frac{1}{\hat{s}^{2}}[\Delta]|\mathcal{M}|^{2} \frac{d^{2} k_{t} \mathrm{~d} \alpha}{\alpha(1-\alpha)} \delta\left(M^{2}-\frac{k_{t}^{2}}{\alpha(1-\alpha)}\right) .
$$

Comparison with Eqs. (5),(6) yields the diffractive structure functions

$$
\begin{aligned}
F_{T}^{D}\left(\beta, Q^{2}, x_{I P}, t\right) & =\frac{Q^{4}}{4 \pi^{2} x \alpha_{e m}} \frac{\mathrm{d} \sigma_{T}^{\gamma^{*} p}}{\mathrm{~d} t \mathrm{~d} M^{2}}, \\
\beta g_{1}^{D}\left(\beta, Q^{2}, x_{I P}, t\right) & =\frac{Q^{4}}{16 \pi^{2} x \alpha_{e m}} \frac{\mathrm{d} \Delta \sigma^{\gamma^{*} p}}{\mathrm{~d} t \mathrm{~d} M^{2}} .
\end{aligned}
$$


In the following, we shall only consider the situation of forward diffraction, i.e. $t=0$. Moreover, we work in the high energy $(\operatorname{small}-x)$ limit $\hat{s} \rightarrow \infty$, thus keeping only the leading power in $\hat{s}$.

The diffractive matrix element $\mathcal{M}$ can be decomposed into a perturbatively calculable part for the scattering of the two parton system with the virtual photon and an unintegrated structure function $\phi\left(l_{t}^{2}, x_{I P}\right)$ parametrizing the probability of finding a parton pair with transverse momentum $l_{t}$ and $-l_{t}$ inside the proton. This unintegrated structure function is a non-perturbative object, and a priori unknown. Its integral over $l_{t}^{2}$ generates, at leading logarithmic accuracy, the well-known parton distribution functions:

$$
\begin{aligned}
\int^{\mu^{2}} \mathrm{~d} l_{t}^{2} \phi_{g}\left(l_{t}^{2}, x_{I P}\right) & =x_{I P} g\left(x_{I P}, \mu^{2}\right), \\
\int^{\mu^{2}} \mathrm{~d} l_{t}^{2} \phi_{\Delta g}\left(l_{t}^{2}, x_{I P}\right) & =x_{I P} \Delta g\left(x_{I P}, \mu^{2}\right), \\
\int^{\mu^{2}} \mathrm{~d} l_{t}^{2} \phi_{\Delta q}\left(l_{t}^{2}, x_{I P}\right) & =x_{I P} \Delta q\left(x_{I P}, \mu^{2}\right), \\
\int^{\mu^{2}} \mathrm{~d} l_{t}^{2} \phi_{\Delta \bar{q}}\left(l_{t}^{2}, x_{I P}\right) & =x_{I P} \Delta \bar{q}\left(x_{I P}, \mu^{2}\right) .
\end{aligned}
$$

To investigate the structure of the polarized diffractive matrix element in more detail, it turns out to be convenient to consider first diffraction off a quark target, where the full matrix element can be calculated perturbatively. In this case, the unintegrated structure functions reduce to simple perturbative splitting functions. The relation between quark and proton matrix elements is given by the replacement

$$
\frac{\alpha_{s}}{2 \pi} \lim _{x_{I P} \rightarrow 0}\left(x_{I P} P_{i q}\left(x_{I P}\right)\right) \int \frac{\mathrm{d} l_{t}^{2}}{l_{t}^{2}} \longrightarrow \int \mathrm{d} l_{t}^{2} \phi_{i}\left(l_{t}^{2}, x_{I P}\right) .
$$

In unpolarized diffraction, only the two gluon exchange amplitude, Fig. 1.a, yields a dominant contribution, the quark-antiquark amplitude, Fig. 1.b, is suppressed by one power of $x$. In the polarized case, both amplitudes contribute with the same powers in $x$, as be shall demonstrate by explicit calculation below.

To study the helicity structure of the diffractive exchange, let us first consider the square of the two gluon exchange amplitude, Fig. 1.a. Taking the target trace for an unpolarized/polarized quark target, one finds in the high energy limit

$$
\begin{aligned}
\operatorname{Tr}\left(\not p \gamma^{\mu_{2}}(\not p-\not / 2) \gamma^{\nu_{2}} \not p^{\prime} \gamma^{\nu_{1}}(\not p-\not / 1) \gamma^{\mu_{1}}\right)= & 32 p^{\mu_{1}} p^{\nu_{1}} p^{\mu_{2}} p^{\nu_{2}} \\
\operatorname{Tr}\left(\gamma_{5} \not p \gamma^{\mu_{2}}(\not p-\not / 2) \gamma^{\nu_{2}} \not p^{\prime} \gamma^{\nu_{1}}(\not p-\not / 1) \gamma^{\mu_{1}}\right)= & 16 i\left(p^{\mu_{1}} p^{\nu_{1}} \epsilon^{\mu_{2} \nu_{2} \rho \sigma} p_{\rho} l_{2, \sigma}\right. \\
& \left.-p^{\mu_{2}} p^{\nu_{2}} \epsilon^{\mu_{1} \nu_{1} \rho \sigma} p_{\rho} l_{1, \sigma}\right) .
\end{aligned}
$$

Contraction of these formulae with the $t$-channel gluon propagators

$$
-g^{\alpha \alpha^{\prime}}=-g_{t}^{\alpha \alpha^{\prime}}+\frac{p^{\alpha} q^{\prime \alpha^{\prime}}+p^{\alpha^{\prime}} q^{\prime \alpha}}{p \cdot q^{\prime}}
$$



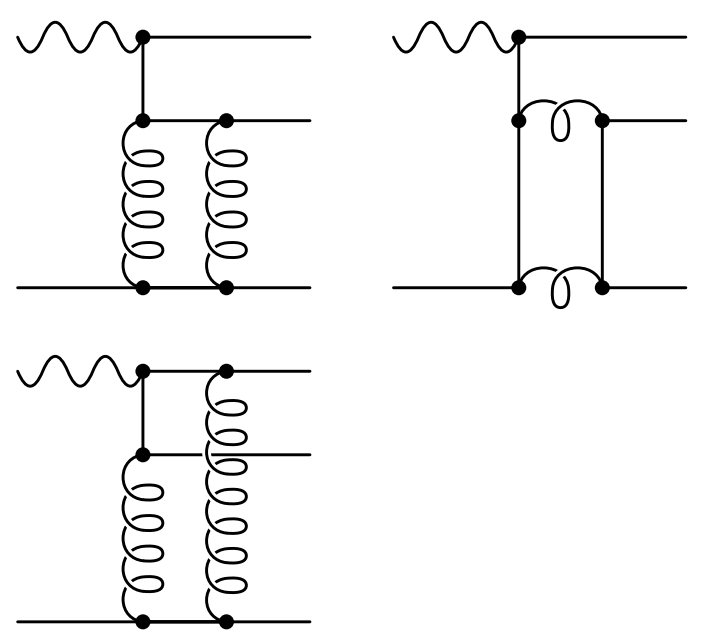

(a)

(b)

Figure 1: Partonic processes contributing to diffractive $q \bar{q}$ production off a quark target: (a) $t$-channel gluon exchange, (b) $t$-channel quark exchange.

shows that all exchanged gluons in the unpolarized $|\mathcal{M}|^{2}$ carry longitudinal polarization, in the polarized $\Delta|\mathcal{M}|^{2}$ only one of the gluons is transversely polarized. The factorization of the polarized target trace into the structures $p^{\mu_{i}} p^{\nu_{i}}$ and $\epsilon^{\mu_{j} \nu_{j} \rho \sigma} p_{\rho} l_{j, \sigma}$ illustrates moreover that the polarized $\Delta|\mathcal{M}|^{2}$ is the product of an amplitude with polarized gluon exchange with the well known unpolarized amplitude, as first pointed out in [6].

The matrix elements for the process $\gamma^{*} q \rightarrow q q \bar{q}$ can be written in a form which makes this factorization explicit:

$$
\begin{array}{rl}
|\mathcal{M}|^{2}=\sum_{q} R_{q} & \hat{s} \alpha(1-\alpha)\left(\alpha^{2}+(1-\alpha)^{2}\right) \\
\times & \left\{\frac{2 k_{t}^{i}}{\bar{Q}^{2}+k_{t}^{2}}-\frac{\left(k_{t}+l_{t, 1}\right)^{i}}{\bar{Q}^{2}+\left(k_{t}+l_{t, 1}\right)^{2}}-\frac{\left(k_{t}-l_{t, 1}\right)^{i}}{\bar{Q}^{2}+\left(k_{t}-l_{t, 1}\right)^{2}}\right\} \\
\times & \left\{\frac{2 k_{t}^{i}}{\bar{Q}^{2}+k_{t}^{2}}-\frac{\left(k_{t}+l_{t, 2}\right)^{i}}{\bar{Q}^{2}+\left(k_{t}+l_{t, 2}\right)^{2}}-\frac{\left(k_{t}-l_{t, 2}\right)^{i}}{\bar{Q}^{2}+\left(k_{t}-l_{t, 2}\right)^{2}}\right\}, \\
\Delta|\mathcal{M}|_{g}^{2}=-\sum_{q} & 2 R_{q}\left\{(1-2 \alpha)^{2} l_{t, 1}^{2}\left(\frac{2 k_{t}^{i}}{\bar{Q}^{2}+k_{t}^{2}}-\frac{\left(k_{t}+l_{t, 1}\right)^{i}}{\bar{Q}^{2}+\left(k_{t}+l_{t, 1}\right)^{2}}-\frac{\left(k_{t}-l_{t, 1}\right)^{i}}{\bar{Q}^{2}+\left(k_{t}-l_{t, 1}\right)^{2}}\right)\right. \\
+ & 2\left(\alpha^{2}+(1-\alpha)^{2}\right) \\
& \times\left(\frac{1}{\bar{Q}^{2}+\left(k_{t}+l_{t, 1}\right)^{2}}\left(l_{t, 1} \cdot\left(l_{t, 1}+k_{t}\right) k_{t}^{i}-k_{t} \cdot\left(l_{t, 1}+k_{t}\right) l_{t, 1}^{i}\right)\right.
\end{array}
$$




$$
\begin{aligned}
&\left.\left.+\frac{1}{\bar{Q}^{2}+\left(k_{t}-l_{t, 1}\right)^{2}}\left(l_{t, 1} \cdot\left(l_{t, 1}-k_{t}\right) k_{t}^{i}-k_{t} \cdot\left(l_{t, 1}-k_{t}\right) l_{t, 1}^{i}\right)\right)\right\} \\
& \times\left\{\frac{2 k_{t}^{i}}{\bar{Q}^{2}+k_{t}^{2}}-\frac{\left(k_{t}+l_{t, 2}\right)^{i}}{\bar{Q}^{2}+\left(k_{t}+l_{t, 2}\right)^{2}}-\frac{\left(k_{t}-l_{t, 2}\right)^{i}}{\bar{Q}^{2}+\left(k_{t}-l_{t, 2}\right)^{2}}\right\}, \\
& \Delta|\mathcal{M}|_{q}^{2}=-2 R_{q} \frac{C_{F}}{T_{F}} \alpha(1-2 \alpha) l_{t, 1}^{2} \frac{k_{t}^{i}}{\bar{Q}^{2}+k_{t}^{2}} \\
& \times\left\{\frac{2 k_{t}^{i}}{\bar{Q}^{2}+k_{t}^{2}}-\frac{\left(k_{t}+l_{t, 2}\right)^{i}}{\bar{Q}^{2}+\left(k_{t}+l_{t, 2}\right)^{2}}-\frac{\left(k_{t}-l_{t, 2}\right)^{i}}{\bar{Q}^{2}+\left(k_{t}-l_{t, 2}\right)^{2}}\right\}, \\
& \Delta|\mathcal{M}|_{\bar{q}}^{2}=-2 R_{q} \frac{C_{F}}{T_{F}}(1-\alpha)(1-2 \alpha) l_{t, 1}^{2} \frac{k_{t}^{i}}{\bar{Q}^{2}+k_{t}^{2}} \\
& \times\left\{\frac{2 k_{t}^{i}}{\bar{Q}^{2}+k_{t}^{2}}-\frac{\left(k_{t}+l_{t, 2}\right)^{i}}{\bar{Q}^{2}+\left(k_{t}+l_{t, 2}\right)^{2}}-\frac{\left(k_{t}-l_{t, 2}\right)^{i}}{\bar{Q}^{2}+\left(k_{t}-l_{t, 2}\right)^{2}}\right\} .
\end{aligned}
$$

The common factor from couplings, colour structure and from the integration over both t-channel loops reads:

$$
R_{q}=512 \pi \alpha_{s}^{4} \alpha_{e m} e_{q}^{2} \frac{T_{F}^{2} C_{F}^{2}}{N_{c}} \hat{s} \frac{\mathrm{d}^{2} l_{t, 1}}{l_{t, 1}^{4}} \frac{\mathrm{d}^{2} l_{t, 2}}{l_{t, 2}^{4}}
$$

where $T_{F}=1 / 2$ and the $l_{t, i}^{2}$-integrations are bound by $l_{t, i}^{2}<\min (\alpha, 1-\alpha) \hat{s}$ due to the requirement of an on-shell cut across the exchange loop. The subscript $g, q, \bar{q}$ on the polarized $\Delta|M|^{2}$ denotes the nature of the $t$-channel exchange in the polarized amplitude. The antiquark exchange amplitude is obtained by considering diffraction off an antiquark target. Note that the replacement (18) acquires a negative sign if applied to a polarized antiquark target.

The polarized matrix elements are suppressed by one power of $\hat{s}$ with respect to the unpolarized matrix element. This suppression can be understood from the structure of the target trace, eqs. (19), (20). Contraction with the photon trace yields one power of $\hat{s}$ for every occurrence of $p^{\mu}$, while $l_{n}^{\mu}$ can only yield terms of the type $l_{t, n}^{2}$ and $l_{t, n} \cdot k_{t}$. This one extra power of $\hat{s}$ is compensated by $1 / x_{I P}$ appearing in the unpolarized quark-to-gluon splitting function on the right hand side of (18).

Inserting the above results in eqs.(12), (13), one obtains expressions for the perturbative contribution to the diffractive structure function. After carrying out the angular integrals in $\vec{l}_{t, n}$ and applying (18), we find:

$$
\begin{aligned}
F_{T}^{D}\left(\beta, Q^{2}, x_{I P}, t\right) & \left.\right|_{t=0}=\frac{Q^{4}}{x N_{c}} \sum_{q} e_{q}^{2} \int \frac{\mathrm{d} k_{t}^{2}}{k_{t}^{4}} \alpha^{2}(1-\alpha)^{2} \frac{\alpha^{2}+(1-\alpha)^{2}}{|1-2 \alpha|} \\
\times & {\left[\alpha_{s} \int \frac{\mathrm{d} l_{t}^{2}}{4 l_{t}^{2}}\left(\frac{k_{t}^{2}-\bar{Q}^{2}}{k_{t}^{2}+\bar{Q}^{2}}-\frac{k_{t}^{2}-l_{t}^{2}-\bar{Q}^{2}}{\sqrt{\left[\bar{Q}^{2}+k_{t}^{2}+l_{t}^{2}\right]^{2}-4 l_{t}^{2} k_{t}^{2}}}\right) \phi_{g}\left(l_{t}^{2}, x_{I P}\right)\right]^{2}, }
\end{aligned}
$$




$$
\begin{gathered}
\left.\beta g_{1}^{D}\left(\beta, Q^{2}, x_{I P}, t\right)\right|_{t=0}=\frac{\beta Q^{4}}{4 x N_{c}} \sum_{q} e_{q}^{2} \int \frac{\mathrm{d} k_{t}^{2}}{k_{t}^{4}} \alpha^{2}(1-\alpha)^{2} \\
\times\left[-\alpha_{s} 2 T_{F} \int \frac{\mathrm{d} l_{t, 1}^{2}}{2 l_{t, 1}^{2}}\left\{|1-2 \alpha| \frac{l_{t, 1}^{2}}{\bar{Q}^{2}}\left(\frac{k_{t}^{2}-\bar{Q}^{2}}{k_{t}^{2}+\bar{Q}^{2}}-\frac{k_{t}^{2}-l_{t, 1}^{2}-\bar{Q}^{2}}{\sqrt{\left[\bar{Q}^{2}+k_{t}^{2}+l_{t, 1}^{2}\right]^{2}-4 l_{t, 1}^{2} k_{t}^{2}}}\right)\right.\right. \\
\left.+\frac{\alpha^{2}+(1-\alpha)^{2}}{|1-2 \alpha|} \frac{1}{\bar{Q}^{2}}\left(\bar{Q}^{2}+k_{t}^{2}+l_{t, 1}^{2}-\sqrt{\left[\bar{Q}^{2}+k_{t}^{2}+l_{t, 1}^{2}\right]^{2}-4 l_{t, 1}^{2} k_{t}^{2}}\right)\right\} \phi_{\Delta g}\left(l_{t, 1}^{2}, x_{I P}\right) \\
\left.+\alpha_{s}|1-2 \alpha| C_{F} \frac{k_{t}^{2}}{\bar{Q}^{2}\left(\bar{Q}^{2}+k_{t}^{2}\right)} \int \mathrm{d} l_{t, 1}^{2}\left(\phi_{\Delta q}\left(l_{t, 1}^{2}, x_{I P}\right)+\phi_{\Delta \bar{q}}\left(l_{t, 1}^{2}, x_{I P}\right)\right)\right] \\
\times\left[\alpha_{s} \int \frac{\mathrm{d} l_{t, 2}^{2}}{4 l_{t, 2}^{2}}\left(\frac{k_{t}^{2}-\bar{Q}^{2}}{k_{t}^{2}+\bar{Q}^{2}}-\frac{k_{t}^{2}-l_{t, 2}^{2}-\bar{Q}^{2}}{\sqrt{\left[\bar{Q}^{2}+k_{t}^{2}+l_{t, 2}^{2}\right]^{2}-4 l_{t, 2}^{2} k_{t}^{2}}}\right) \phi_{g}\left(l_{t, 2}^{2}, x_{I P}\right)\right], \quad(27)
\end{gathered}
$$

where $\bar{Q}^{2}=\alpha(1-\alpha) Q^{2}$. The parameter $\alpha$ is itself not an independent variable, but related to $k_{t}^{2}=\alpha(1-\alpha) Q^{2}(1-\beta) / \beta$. The result for $F_{T}^{D}$ is in agreement with earlier results in the literature [四, 5].

The integrations over the transverse momenta of the exchanged $\left(l_{t, i}\right)$ and final state $\left(k_{t}\right)$ partons extend into the region of small momenta, where the perturbative calculation is expected to be no longer applicable. A simple model assumption for the infrared behaviour of the unintegrated structure functions $\phi\left(l_{t}^{2}, x_{I P}\right)$, as proposed for the unpolarized diffractive structure function in [7], allows however to extrapolate the expressions obtained above into the region of small $l_{t, i}$ and small $k_{t}$. We will demonstrate below that the resulting expression for $g_{1}^{D}$ turns out to be insensitive on the infrared behaviour of the polarized unintegrated distributions.

Following the argumentation in [7], we introduce a hadronic scale $k_{0}^{2}$, where the transition between soft and hard dynamics takes place. Around this scale, the $l_{t}^{2}$-dependence of $\phi\left(l_{t}^{2}, x_{I P}\right)$ changes from the perturbative $1 / l_{t}^{2}$ behaviour (cf. eq. (18)) to a constant $1 / k_{0}^{2}$ :

$$
\phi\left(l_{t}^{2}, x_{I P}\right) \sim \frac{1}{k_{0}^{2}}\left(\frac{k_{0}^{2}}{l_{t}^{2}}\right)^{\nu\left(l_{t}^{2} / k_{0}^{2}\right)} \quad \text { with } \nu\left(l_{t}^{2} / k_{0}^{2}\right) \longrightarrow\left\{\begin{array}{lll}
1 & : & l_{t}^{2} \gg k_{0}^{2} \\
0 & : & l_{t}^{2} \ll k_{0}^{2}
\end{array}\right.
$$

With this assumption, all $l_{t, i}^{2}$ integrals remain finite, while preserving the leading logarithmic behaviour as given in (14)-(17). The normalization of $\phi\left(l_{t}^{2}, x_{I P}\right)$ in the infrared region is determined entirely by non-perturbative effects and has to be taken as free parameter.

Closer inspection of the $l_{t, i}^{2}$-integrands shows that for large $k_{t}^{2}$ the dominant contribution to the unpolarized amplitude comes from the region $k_{0}^{2}<l_{t, 2}^{2}<k_{t}^{2}+\bar{Q}^{2}$. The polarized gluon induced amplitude also changes its dependence on $l_{t, i}^{2}$ around $l_{t, i}^{2}=k_{t}^{2}+\bar{Q}^{2}$. Unlike the unpolarized amplitude, it does however not acquire an additional suppression factor $1 / l_{t, i}^{2}$ for larger $l_{t, i}^{2}$, such that the relevant integration region for the polarized amplitudes is bound only by the kinematical cut: $k_{0}^{2}<l_{t, 1}^{2}<\min (\alpha, 1-\alpha) \hat{s}$.

These upper cuts on $l_{t, i}^{2}$ determine, at the leading logarithmic level, the scale at which the target structure is probed. It is therefore appropriate to define the hard, perturbative 
contribution to the $k_{t}^{2}$-integral by demanding $k_{t}^{2}+\bar{Q}^{2}>k_{0}^{2}$. This perturbative contribution to $F_{T}^{D}$ can be obtained by retaining only the leading power in $l_{t, n}^{2}$ in the integrands. It takes the well known 4 , 5] form

$$
\begin{aligned}
\left.F_{T, \text { hard }}^{D}\left(\beta, Q^{2}, x_{I P}, t\right)\right|_{t=0}= & \frac{\beta}{x_{I P} N_{c}} \sum_{q} e_{q}^{2} \beta^{2}(1-\beta) \int_{k_{0}^{2}}^{\frac{Q^{2}}{4 \beta}} \frac{\mathrm{d} \tilde{k}^{2}}{\tilde{k}^{4}} \frac{1-2 \beta \tilde{k}^{2} / Q^{2}}{\sqrt{1-4 \beta \tilde{k}^{2} / Q^{2}}} \\
& \times\left[\alpha_{s} \int_{k_{0}^{2}}^{\tilde{k}^{2}} \mathrm{~d} l_{t}^{2} \phi_{g}\left(l_{t}^{2}, x_{I P}\right)\right]^{2},
\end{aligned}
$$

with $\tilde{k}^{2}=k_{t}^{2} /(1-\beta)=k_{t}^{2}+\bar{Q}^{2}$. The hard contribution to $g_{1}^{D}$ consists of two terms, corresponding to the regions with $l_{t, 1}^{2}$ being smaller or larger than $\tilde{k}^{2}$ :

$$
\beta g_{1, \text { hard }}^{D}=\beta g_{1,<}^{D}+\beta g_{1,>}^{D}
$$

These terms read:

$$
\begin{aligned}
&\left.\beta g_{1,<}^{D}\left(\beta, Q^{2}, x_{I P}, t\right)\right|_{t=0}= \frac{\beta}{4 x_{I P} N_{c}} \sum_{q} e_{q}^{2} \beta(1-\beta) \int_{k_{0}^{2}}^{\frac{Q^{2}}{4 \beta}} \frac{\mathrm{d} \tilde{k}^{2}}{\tilde{k}^{4}} \\
& \times {\left[\alpha _ { s } \int _ { k _ { 0 } ^ { 2 } } ^ { \tilde { k } ^ { 2 } } \mathrm { d } l _ { t , 1 } ^ { 2 } \left(-2 T_{F} \frac{1-2 \beta \tilde{k}^{2} / Q^{2}}{\sqrt{1-4 \beta \tilde{k}^{2} / Q^{2}}} \phi_{\Delta g}\left(l_{t, 1}^{2}, x_{I P}\right)\right.\right.} \\
&\left.\left.+C_{F} \sqrt{1-4 \beta \tilde{k}^{2} / Q^{2}}\left(\phi_{\Delta_{q}}\left(l_{t, 1}^{2}, x_{I P}\right)+\phi_{\Delta_{\bar{q}}}\left(l_{t, 1}^{2}, x_{I P}\right)\right)\right)\right] \\
& \times\left[\alpha_{s} \int_{k_{0}^{2}}^{\tilde{k}^{2}} \mathrm{~d} l_{t, 2}^{2} \phi_{g}\left(l_{t, 2}^{2}, x_{I P}\right)\right] \\
&\left.\beta g_{1,>}^{D}\left(\beta, Q^{2}, x_{I P}, t\right)\right|_{t=0}= \frac{\beta}{4 x_{I P} N_{c}} \sum_{q} e_{q}^{2} \beta(1-\beta) \int_{k_{0}^{2}}^{\frac{Q^{2}}{4 \beta}} \frac{\mathrm{d} \tilde{k}^{2}}{\tilde{k}^{4}} \sqrt{1-4 \beta \tilde{k}^{2} / Q^{2}} \\
& \times\left[\alpha_{s} \int_{\tilde{k}^{2}}^{\tilde{k}^{2} / x_{I P}} \mathrm{~d} l_{t, 1}^{2}\left(-2 T_{F} \phi_{\Delta g}\left(l_{t, 1}^{2}, \widehat{x_{I P}}\right)+C_{F}\left(\phi_{\Delta_{q}}\left(l_{t, 1}^{2}, \widehat{x_{I P}}\right)+\phi_{\Delta_{\bar{q}}}\left(l_{t, 1}^{2}, \widehat{x_{I P}}\right)\right)\right)\right] \\
& \quad\left[\alpha_{s} \int_{k_{0}^{2}}^{\tilde{k}^{2}} \mathrm{~d} l_{t, 2}^{2} \phi_{g}\left(l_{t, 2}^{2}, x_{I P}\right)\right] .
\end{aligned}
$$

It is worth noting that $g_{1,>}^{D}$ corresponds to a region in phase space that is usually discarded in calculations based on strong ordering in transverse momentum. Similar terms are also present in the inclusive polarized structure function $g_{1}$ at small $x$ [2], where they yield double logarithms of the form $\alpha_{s}^{n} \ln ^{2 n}(1 / x)$. These logarithmic terms are potentially large at small $x$ (or small $x_{I P}$ in diffraction). A resummation of them, following closely the $g_{1}$ calculation of [2], will be outlined in section 4.

Note in (32) the $\widehat{x_{I P}}$ used as the argument of parton distributions $\phi_{\Delta j}(j=g, q, \bar{q})$. This argument follows from the on-shell condition across the $t$-channel cut of the diffractive 
amplitude. In the region of $l_{t}^{2} \gg \tilde{k}^{2}$, an energy fraction $x_{I P}+l_{t}^{2} /(\alpha \hat{s})$ or $x_{I P}+l_{t}^{2} /((1-\alpha) \hat{s})$ is needed to fulfill this condition. To implement this energy shift consistently, we have to go back to (23), (24) and can no longer sum up the terms corresponding to the quark-proton centre-of-mass energy $s_{q p}=\alpha \hat{s}$ and $s_{q p}=(1-\alpha) \hat{s}$. Instead of $\phi_{\Delta_{q}}\left(l_{t}^{2}, x_{I P}\right)+\phi_{\Delta_{\bar{q}}}\left(l_{t}^{2}, x_{I P}\right)$ and $\phi_{\Delta_{g}}\left(l_{t}^{2}, x_{I P}\right)$, one finds

$$
\begin{aligned}
\phi_{\Delta_{q}}\left(l_{t}^{2}, \widehat{x_{I P}}\right)+\phi_{\Delta_{\bar{q}}}\left(l_{t}^{2}, \widehat{x_{I P}}\right)= & \frac{1}{2}\left(\phi_{\Delta_{q}}\left(l_{t}^{2}, x_{I P}+\frac{l_{t}^{2}}{\alpha_{-} \hat{s}}\right)+\phi_{\Delta_{q}}\left(l_{t}^{2}, x_{I P}+\frac{l_{t}^{2}}{\alpha_{+} \hat{s}}\right)\right. \\
& \left.+\phi_{\Delta_{\bar{q}}}\left(l_{t}^{2}, x_{I P}+\frac{l_{t}^{2}}{\alpha_{-} \hat{s}}\right)+\phi_{\Delta_{\bar{q}}}\left(l_{t}^{2}, x_{I P}+\frac{l_{t}^{2}}{\alpha_{+} \hat{s}}\right)\right) \\
+\frac{1}{2 \sqrt{1-4 \beta \tilde{k}^{2} / Q^{2}}}\left(-\phi_{\Delta_{q}}\left(l_{t}^{2}, x_{I P}+\frac{l_{t}^{2}}{\alpha_{-} \hat{s}}\right)+\phi_{\Delta_{q}}\left(l_{t}^{2}, x_{I P}+\frac{l_{t}^{2}}{\alpha_{+} \hat{s}}\right)\right. & \left.-\phi_{\Delta_{\bar{q}}}\left(l_{t}^{2}, x_{I P}+\frac{l_{t}^{2}}{\alpha_{-} \hat{s}}\right)+\phi_{\Delta_{\bar{q}}}\left(l_{t}^{2}, x_{I P}+\frac{l_{t}^{2}}{\alpha_{+} \hat{s}}\right)\right), \\
\phi_{\Delta_{g}}\left(l_{t}^{2}, \widehat{x_{I P}}\right)= & \frac{1}{2}\left(\phi_{\Delta_{g}}\left(l_{t}^{2}, x_{I P}+\frac{l_{t}^{2}}{\alpha_{-} \hat{s}}\right)+\phi_{\Delta_{g}}\left(l_{t}^{2}, x_{I P}+\frac{l_{t}^{2}}{\alpha_{+} \hat{s}}\right)\right),
\end{aligned}
$$

where $\alpha_{ \pm}=1 / 2\left(1 \pm \sqrt{1-4 \beta \tilde{k}^{2} / Q^{2}}\right)$. This effect will be taken into account in the next section when we discuss the resummation of the double logarithms.

Up to now, we have discussed the hard, perturbative contribution to the diffractive structure functions. These can be probed only by restricting the diffractive final state such that a minimum cut on $\tilde{k}^{2}$ is realized, for example by demanding a pair of high transverse momentum jets. The inclusive structure function also receives contributions from the soft, non-perturbative region $\tilde{k}^{2}<k_{0}^{2}$. These can not be calculated from first principles; using the model assumption (28) for the infrared behaviour of the unintegrated structure functions, it is however possible to extend (26) and (27) into the soft region. This procedure will enable us to estimate the $\beta$-dependence of the soft contribution to $g_{1}^{D}$, the absolute normalization of this contribution is however determined entirely by non-perturbative effects and cannot be calculated within our approach.

To facilitate the discussion of the soft contributions, let us rewrite (26) and (27) by introducing amplitude functions $\psi_{i}\left(\alpha, k_{t}^{2}, l_{t}^{2}\right)$ :

$$
\begin{aligned}
\left.F_{T}^{D}\left(\beta, Q^{2}, x_{I P}, t\right)\right|_{t=0}= & \frac{1}{x_{I P} N_{c}} \sum_{q} e_{q}^{2} \beta \int \frac{\mathrm{d} \tilde{k}^{2}}{(1-\beta)} \\
& \times\left[\alpha_{s} \int \frac{\mathrm{d} l_{t}^{2}}{l_{t}^{2}} \psi_{g}\left(\alpha, k_{t}^{2}, l_{t}^{2}\right) \phi_{g}\left(l_{t}^{2}, x_{I P}\right)\right]^{2}, \\
\left.\beta g_{1}^{D}\left(\beta, Q^{2}, x_{I P}, t\right)\right|_{t=0}= & \frac{1}{4 x_{I P} N_{c}} \sum_{q} e_{q}^{2} \beta^{2} \int \frac{\mathrm{d} \tilde{k}^{2}}{(1-\beta)} \\
& \times\left[\alpha _ { s } \int \frac { \mathrm { d } l _ { t , 1 } ^ { 2 } } { l _ { t , 1 } ^ { 2 } } \left\{\psi_{\Delta g}\left(\alpha, k_{t}^{2}, l_{t, 1}^{2}\right) \phi_{\Delta g}\left(l_{t, 1}^{2}, x_{I P}\right)\right.\right.
\end{aligned}
$$




$$
\begin{aligned}
& \left.\left.\quad+\psi_{\Delta q}\left(\alpha, k_{t}^{2}, l_{t, 1}^{2}\right) \phi_{\Delta q}\left(l_{t, 1}^{2}, x_{I P}\right)+\psi_{\Delta \bar{q}}\left(\alpha, k_{t}^{2}, l_{t, 1}^{2}\right) \phi_{\Delta \bar{q}}\left(l_{t, 1}^{2}, x_{I P}\right)\right\}\right] \\
& \times\left[\alpha_{s} \int \frac{\mathrm{d} l_{t, 2}^{2}}{l_{t, 2}^{2}} \psi_{g}\left(\alpha, k_{t}^{2}, l_{t, 2}^{2}\right) \phi_{g}\left(l_{t, i}^{2}, x_{I P}\right)\right] .
\end{aligned}
$$

We already discussed the behaviour of the amplitude functions in the context of the perturbative contributions to $g_{1}^{D}$ and $F_{T}^{D}$. For small $\tilde{k}^{2}$, they become:

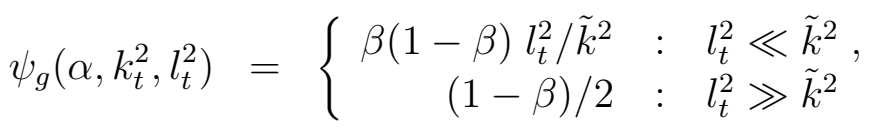

$$
\begin{aligned}
& \psi_{\Delta g}\left(\alpha, k_{t}^{2}, l_{t}^{2}\right)=-\frac{2 T_{F}}{C_{F}} \psi_{\Delta q, \bar{q}}\left(\alpha, k_{t}^{2}, l_{t}^{2}\right) \\
& =-2 T_{F} \frac{1-\beta}{\beta} \frac{l_{t}^{2}}{\tilde{k}^{2}} \quad: \quad \text { all } \quad l_{t}^{2} \text {. }
\end{aligned}
$$

These simple forms allow us to identify the dominant regions in the $l_{t, i}^{2}$ and $\tilde{k}^{2}$ integrations by mere power counting.

In the region of $\tilde{k}^{2}<k_{0}^{2}$, the dominant contribution to the $l_{t, i}^{2}$-integral for the unpolarized amplitude comes from $\tilde{k}^{2}<l_{t, i}^{2}<k_{0}^{2}$ :

$$
\int_{\tilde{k}^{2}}^{k_{0}^{2}} \frac{\mathrm{d} l_{t, i}^{2}}{l_{t, i}^{2}} \psi_{g}\left(\alpha, k_{t}^{2}, l_{t, i}^{2}\right) \phi_{g}\left(l_{t, i}^{2}, x_{I P}\right) \sim(1-\beta) \frac{1}{k_{0}^{2}} \ln \frac{k_{0}^{2}}{\tilde{k}^{2}} .
$$

For the polarized amplitude one finds that, even for $\tilde{k}^{2}<k_{0}^{2}$, the $l_{t, i}^{2}$-integral is dominated by the perturbative region $k_{0}^{2}<l_{t, i}^{2}<\tilde{k}^{2} / x_{I P}$. The non-perturbative behaviour of $\phi_{\Delta g, \Delta q, \Delta \bar{q}}$ becomes relevant only if $\tilde{k}^{2}<x_{I P} k_{0}^{2}$. The contribution from this latter region is however suppressed by a factor $1 / \ln ^{2} x_{I P}$ with respect to the contribution from the former region. The soft contribution to $\beta g_{1}^{D}$ is thus given simply by extrapolating (32) into the region $x_{I P} k_{0}^{2}<\tilde{k}^{2}<k_{0}^{2}$.

However, some caution is to be taken, since this procedure extrapolates the amplitudes into a region of phase space corresponding to transverse momenta of the order $\Lambda_{\mathrm{QCD}}$. Although the polarized amplitudes are (in contrast to the unpolarized amplitude) well behaved in this region, they may still be changed due to parton motion or confinement. Such effects can easily modify the right hand side of (38), where $k_{0}^{2}$ and $\tilde{k}^{2}$ could be accompanied by terms of order $\Lambda_{\mathrm{QCD}}$, thus imposing a natural limit on the accuracy of the soft interpolation.

\section{Resummation of $\ln ^{2}\left(1 / x_{I P}\right)$}

Perturbative QCD corrections to the polarized inclusive structure function $g_{1}\left(x, Q^{2}\right)$ at small $x$ contain leading double logarithmic terms of the form $\alpha_{s}^{n} \ln ^{2 n}(1 / x)$. In spacelike diffractive processes, such terms can only appear in polarized structure functions; the most 
singular terms in the unpolarized singlet case take the form $\alpha_{s}^{n} \ln ^{n}(1 / x)$. The $\alpha_{s}^{n} \ln ^{2 n}(1 / x)$ terms arise from regions in phase space which are discarded in the conventional strong ordering in transverse momentum. A resummation of the leading double logarithmic terms in $g_{1}\left(x, Q^{2}\right)$ at small $x$ has been performed in [2], using an infrared evolution equation [9] for the polarized exchange amplitudes.

From the spin dependence of the target trace (20), one would expect similar exchange amplitudes also to appear in $g_{1}^{D}$. In fact, the occurrence of terms from non-strongly ordered regions in (32) is a first manifestation of a double logarithmic enhancement already at the leading order in $\alpha_{s}$. In the following, we shall briefly outline how these terms can be resummed to all orders. To facilitate the discussion, we shall assume that the polarized distributions for all quark flavours are identical at small $x$, i.e. we will only consider the singlet quark distribution. A resummation of non-singlet contributions would follow exactly the same procedure as outlined below.

Let us consider the combination of unintegrated polarized distributions appearing in (32).1

$$
\begin{aligned}
& \sum_{q} e_{q}^{2} \int^{\mu^{2}} \mathrm{~d} l_{t}^{2}\left[-2 T_{F} \phi_{\Delta g}\left(l_{t}^{2}, x_{I P}\right)+C_{F}\left(\phi_{\Delta_{q}}\left(l_{t}^{2}, x_{I P}\right)+\phi_{\Delta_{\bar{q}}}\left(l_{t}^{2}, x_{I P}\right)\right)\right] \\
& =\sum_{q} \frac{e_{q}^{2}}{n_{f}}(1,0)\left(\begin{array}{cc}
C_{F} & -2 T_{F} n_{f} \\
2 C_{F} & 4 C_{A}
\end{array}\right) \int^{\mu^{2}} \mathrm{~d} l_{t}^{2}\left(\begin{array}{c}
\phi_{\Delta \Sigma}\left(x_{I P}, l_{t}^{2}\right) \\
\phi_{\Delta g}\left(x_{I P}, l_{t}^{2}\right)
\end{array}\right) \\
& \equiv \sum_{q} \frac{e_{q}^{2}}{n_{f}}(1,0) T^{L O}\left(x_{I P}, \mu^{2}, \mu_{0}^{2}\right) \int^{\mu_{0}^{2}} \mathrm{~d} l_{t}^{2}\left(\begin{array}{c}
\phi_{\Delta \Sigma}\left(x_{I P}, l_{t}^{2}\right) \\
\phi_{\Delta g}\left(x_{I P}, l_{t}^{2}\right)
\end{array}\right)
\end{aligned}
$$

where $n_{f}$ is the number of active flavours and $\Delta \Sigma=\sum_{q}(\Delta q+\Delta \bar{q})$. The above equation coincides with the contribution from the first rung of an ordinary DGLAP ladder amplitude (evolved from given boundary conditions at $\mu_{0}^{2}$ to larger $\mu^{2}$ ) to the inclusive structure function $g_{1}\left(x, Q^{2}\right)$ in the small- $x$ limit [8]. In addition to this term, (31) also contains

$$
\begin{aligned}
& -\sum_{q} e_{q}^{2} 2 T_{F} \int^{\mu^{2}} \mathrm{~d} l_{t}^{2} \phi_{\Delta g}\left(l_{t}^{2}, x_{I P}\right) \\
& \quad=\sum_{q} \frac{e_{q}^{2}}{n_{f}} \frac{T_{F} n_{f}}{2\left(T_{F} n_{f}+C_{A}\right)}(2,-1) T^{L O}\left(x_{I P}, \mu^{2}, \mu_{0}^{2}\right) \int^{\mu_{0}^{2}} \mathrm{~d} l_{t}^{2}\left(\begin{array}{c}
\phi_{\Delta \Sigma}\left(x_{I P}, l_{t}^{2}\right) \\
\phi_{\Delta g}\left(x_{I P}, l_{t}^{2}\right)
\end{array}\right) .
\end{aligned}
$$

Resummation of the double leading logarithmic contributions turns $T^{L O}$ into a resummed evolution matrix $T$.

This evolution matrix can be expressed by its Mellin transformation $R\left(\omega, \mu^{2}, \mu_{0}^{2}\right)$ as

$$
T\left(x_{I P}, \mu^{2}, \mu_{0}^{2}\right)=\int_{-i \infty}^{+i \infty} \frac{\mathrm{d} \omega}{2 \pi i}\left(\frac{s_{q p}}{\mu^{2}}\right)^{\omega} \frac{e^{-i \pi \omega}-1}{2} R\left(\omega, \mu^{2}, \mu_{0}^{2}\right) .
$$

\footnotetext{
${ }^{1}$ Note that the notation used here differs from [2]. The vectors $T$ and $R$ used there are obtained from our matrices $T$ and $R$ by contraction with $\left(2 e_{q}^{2}, 0\right)$. Furthermore, all evolution matrices are transposed compared to [2].
} 
The matrix $R$ obeys an infrared evolution equation, given by (3.7) in [2]:

$$
\left(\omega-\frac{\partial}{\partial \ln \mu^{2}}\right) R=\frac{1}{8 \pi^{2}} F_{0} R
$$

where the evolution kernel matrix $F_{0}$ contains all partonic colour singlet $t$-channel exchanges at small $x . F_{0}$ is itself determined by an evolution equation for four parton amplitudes at small $x$, as calculated in [2].

The matrix $F_{0}$ can be diagonalized to

$$
\hat{F}_{0}=\operatorname{diag}\left(\lambda_{0}^{+}, \lambda_{0}^{-}\right) \quad \text { with } \quad F_{0}=E_{0}^{-1} \hat{F}_{0} E_{0}
$$

where $E_{0}$ is the matrix of eigenvectors of $F_{0}$. Using these, we can solve the evolution equation (42):

$$
R\left(\omega, \mu^{2}, \mu_{0}^{2}\right)=E_{0}^{-1} \frac{1}{\omega-\hat{F}_{0} /\left(8 \pi^{2}\right)}\left(\frac{\mu^{2}}{\mu_{0}^{2}}\right)^{\hat{F}_{0} /(8 \pi)} E_{0}\left(\begin{array}{c}
2 e_{q}^{2} \\
0
\end{array}\right)
$$

transformation according to (41) then yields the resummed evolution matrix $T$. Some numerical studies on $R$ can be found in [2].

The resummed expression for $g_{1}^{D}$ is obtained by introducing the vectors

$$
T^{(a)}=(1,0) T, \quad T^{(b)}=\frac{T_{F} n_{f}}{2\left(T_{F} n_{f}+C_{A}\right)}(2,-1) T
$$

and replacing the integrals over the unintegrated structure functions in (31) and (32) by the corresponding resummed expressions. $T^{(a)}$ and $T^{(b)}$ should be considered as the sum of two terms corresponding to the quark energies $s_{q p}=\alpha \hat{s}$ and $s_{q p}=(1-\alpha) \hat{s}$, as listed in $(33)$.

Recall that in the double logarithmic approximation the negative signature amplitude sums up not only the ladder graphs but also nonladder diagrams with transverse momenta of the embraced gluons $q_{t}^{2}>\mu^{2}$ as well [9]. For $\mu^{2}>k_{t}^{2}$ all such contributions are already included into the amplitude $T$ (41). The presence of the spectator quark in the diffractive amplitude could in principle yield additional nonladder contributions. In these contributions, the large gluon momentum $q_{t}$ has however to appear in two quark propagators, such that these terms do not bear a leading logarithm. Thus the presence of the spectator quark does not destroy the structure of the double logarithmic amplitudes.

The perturbative contributions from different regions in $l_{t, 1}^{2}$ can moreover be combined into a single expression. The resulting double leading logarithmic expressions read:

$$
\begin{aligned}
& \left.\beta g_{1, \text { hard,DLL }}^{D}\left(\beta, Q^{2}, x_{I P}, t\right)\right|_{t=0}=\frac{\beta}{4 x_{I P} N_{c}} \sum_{q} e_{q}^{2} \beta(1-\beta) \int_{k_{0}^{2}}^{\frac{Q^{2}}{4 \beta}} \frac{\mathrm{d} \tilde{k}^{2}}{\tilde{k}^{4}} \sqrt{1-4 \beta \tilde{k}^{2} / Q^{2}} \\
& \times\left[\alpha_{s}\left(T^{(a)}\left(x_{I P}, \tilde{k}^{2} / x_{I P}, k_{0}^{2}\right)+\frac{2 \beta \tilde{k}^{2} / Q^{2}}{1-4 \beta \tilde{k}^{2} / Q^{2}} T^{(b)}\left(x_{I P}, \tilde{k}^{2}, k_{0}^{2}\right)\right)\left(\begin{array}{c}
\Delta \Sigma\left(x_{I P}, k_{0}^{2}\right) \\
\Delta g\left(x_{I P}, k_{0}^{2}\right)
\end{array}\right)\right]
\end{aligned}
$$




$$
\begin{aligned}
& \times\left[\alpha_{s} \int_{k_{0}^{2}}^{\tilde{k}^{2}} \mathrm{~d} l_{t, 2}^{2} \phi_{g}\left(l_{t, 2}^{2}, x_{I P}\right)\right] \\
&\left.\beta g_{1, \mathrm{soft}, \mathrm{DLL}}^{D}\left(\beta, Q^{2}, x_{I P}, t\right)\right|_{t=0}=\frac{\beta}{8 x_{I P} N_{c}} \sum_{q} e_{q}^{2}(1-\beta) \int_{x_{I P} k_{0}^{2}}^{k_{0}^{2}} \frac{\mathrm{d} \tilde{k}^{2}}{\tilde{k}^{2}} \\
& \times\left[\alpha_{s} T^{(a)}\left(x_{I P}, \tilde{k}^{2} / x_{I P}, k_{0}^{2}\right)\left(\begin{array}{c}
\Delta \Sigma\left(x_{I P}, k_{0}^{2}\right) \\
\Delta g\left(x_{I P}, k_{0}^{2}\right)
\end{array}\right)\right] \\
& \times\left[\alpha_{s} \int_{\tilde{k}^{2}}^{k_{0}^{2}} \frac{\mathrm{d} l_{t, 2}^{2}}{l_{t, 2}^{2}} \phi_{g}\left(l_{t, 2}^{2}, x_{I P}\right)\right] .
\end{aligned}
$$

It is evident from the above expressions, that contributions containing $T^{(b)}$ appear only for large $\tilde{k}^{2}$, which is realized e.g. in diffractive jet production. For most other observables, such as for example the diffractive structure function itself, or diffractive vector meson production [10, 6], this term plays only a minor role. In these latter cases, the resummed diffractive amplitude becomes directly proportional to the inclusive polarized structure function at small $x$.

\section{Conclusions and Outlook}

In this paper we have calculated the longitudinal spin-spin asymmetry for the heavy photon to $q \bar{q}$-pair diffractive dissociation in the framework of a perturbative two parton exchange model. The spin asymmetry is given by the interference between unpolarized and polarized diffractive amplitudes. The spin dependent amplitude has a double logarithmic form and includes the contribution from the region of inverse $k_{t}$ ordering where the transverse momentum of the $t$-channel parton $l_{t}$ is larger than the transverse momentum $k_{t}$ of the outgoing quark. We provide explicit expressions for strongly ordered and inversely ordered contributions to the diffractive structure function $g_{1}^{D}$ in terms of unintegrated structure functions. $g_{1}^{D}$ receives contributions from both polarized quark and gluon distributions at small $x$. Like in the inclusive structure function $g_{1}$, a positive $\Delta g$ at small $x$ results in a negative diffractive spin asymmetry.

We present an expression which provides the full summation of all double logarithmic contributions in the spin dependent amplitude at small $x$. The expression can smoothly be continued into the infrared region and matched with the soft part of the amplitude.

Our results show that the diffractive asymmetry increases towards small $\beta: A^{D} \sim$ $1 / \beta$. We must however emphasize that in this paper only the $\gamma^{*} \rightarrow q \bar{q}$ dissociation is considered. To avoid contributions from more complicated final states our results for the asymmetry should be used only in the large- $\beta$ domain $\left(\beta>0.3\right.$, i.e. $\left.M^{2}>2 Q^{2}\right)$ where the contamination of $q \bar{q}+g$ states is presumably small.

A numerical estimate of the asymmetry can be obtained from eq. (29) and (31) by approximating the unintegrated parton distributions at leading $\ln Q^{2}$, (14)-(17). Using recent parametrizations of unpolarized and polarized parton distributions, one finds a resulting asymmetry $A_{L}$ of the order $-10^{-2} \ldots-10^{-3}$ at $Q^{2} \sim 10 \mathrm{GeV}^{2}$ and $x_{I P} \sim 10^{-3}$, which is at least an order of magnitude above the non-perturbative estimates of [1]. The 
estimated asymmetry is moreover rather insensitive on the precise value used for the matching scale $k_{0}^{2}$. It must however be kept in mind that this estimate relies on the behaviour of the polarized gluon distribution at small $x$, which is not known directly from experiment at present, but can be at best inferred indirectly from the observed evolution of the polarized structure function. Like in the inclusive structure function $g_{1}$ [11], the resummation of $\ln ^{2}\left(1 / x_{I P}\right)$ will result in a substantial enhancement of the asymmetry.

\section{Acknowledgements}

The work of MGR was supported by the Russian Fund of Fundamental Research (98 02 17629), the INTAS grant 95 - 311, by the Volkswagen-Stiftung and by DESY. JB was supported in part by the EU Fourth Framework Programme 'Training and Mobility of Researchers', Network 'Quantum Chromodynamics and the Deep Structure of Elementary Particles', contract FMRX-CT98-0194 (DG 12 - MIHT).

\section{References}

[1] S.I. Manaenkov, preprint DESY 99-016 (hep-ph/9903405).

[2] J. Bartels, B. Ermolaev and M.G. Ryskin, Z. Phys. C70 (1996) 273; C72 (1996) 627.

[3] B. Badelek et al., J. Phys. G22 (1996) 815.

[4] M.G. Ryskin and M. Besancon, Proceedings of the Workshop "Physics at HERA", eds. W. Buchmüller and G. Ingelman, DESY (Hamburg, 1991), p.215;

E. Levin and M. Wüsthoff, Phys. Rev. D50 (1994) 4306;

N.N. Nikolaev and B.G. Zakharov, Phys. Lett. B332 (1994) 177;

J. Bartels, H. Lotter, and M. Wüsthoff, Phys.Lett. B379 (1996) 239; Erratum ibid.B382 (1996) 449.

[5] M. Wüsthoff, Phys. Rev. D56 (1997) 4311.

[6] M.G. Ryskin, Phys. Atom. Nucl. 62 (1999) 315 [Yad. Fiz. 62 (1999) 350].

[7] J. Bartels, J. Ellis, H. Kowalski and M. Wüsthoff, Eur. Phys. J. C7 (1999) 443.

[8] M.A. Ahmed and G.G. Ross, Phys. Lett. 56B (1975) 385; Nucl. Phys. B111 (1976) 441.

[9] R. Kirschner and L.N. Lipatov, Nucl. Phys. B213 (1983) 122.

[10] A.D. Martin, M.G. Ryskin and T. Teubner, Phys. Rev. D55 (1997) 4329, ibid. D56 (1997) 3007.

[11] J. Kwiecinski, B. Ziaja, preprint hep-ph/9902440. 